\title{
The LHC Experimental Programme
}

\author{
K. Eggert and W. Hoogland
}

\author{
CERN, Geneva, Switzerland
}

Crucial to our understanding of one of the most fundamental problems in particle physics - the origin of electroweak symmetry breaking - will be to cross the 1 Tev frontier for constituent collisions at the Large Hadron Collider (LHC) proposed as CERN's next accelerator facility. In making extensive use of existing infrastructure - the Large Electron Proton (LEP) collider tunnel and other parts of CERN's accelerator complex - the LHC will be an extremely cost-effective way to provide several unique but complementary physics options up to energies of almost $16 \mathrm{TeV}$ and luminosities exceeding $10^{34} / \mathrm{cm}^{2} \mathrm{~s}$. LHC will extend CERN's tradition of providing its user community with state-of-the-art facilities which would span a broad diversified physics programme well into the next century.

\section{Physics Programme}

The physics programme for the LHC is very rich for there are many interesting aspects to experiments in the new energy range of up to a few $\mathrm{TeV}$ in constituentconstituent collisions (Fig. 1). These aspects could provide answers to some of the key questions within and beyond the Standard Model of particle physics. In particular, the LHC would certainly elucidate the mass problem.

All confirmed experimental results in particle physics continue to agree with the Standard Model - the electroweak unification of electromagnetism and the weak nuclear force coupled with the quantum chromodynamics (QCD) field theory of quark interactions. LEP experiments have tested the Standard Model to a fraction of a percent and have shown the existence of just three quark-lepton generations.

Despite its success, the Standard Model is not the ultimate fundamental theory: there are manifest inadequacies pointing to new physics. Three classes of unsolved problems stand out in particular, namely those of mass (what are the origins of the different particle masses and their observed magnitudes?); of flavour (why are there three generations of quarks and leptons - as established by LEP - and what are the origins of CP-violation and of the charged weak-current mixing

Karsten Eggert, who teaches at the TH Aachen in Germany, is reponsible for physics coordination in CERN's Accelerator Technology Division.

Walter Hoogland, Professor of Particle Physics at the University of Amsterdam, is the Director of Research at CERN, CH-1211 Geneva 23. angles?); and of unification (are the various interactions we see, including gravity, just different manifestations of the same underlying fundamental force?). There are also likely to be unexpected phenomena that cast these problems in a novel light or raise new concerns.

\section{Higgs boson}

The most pressing issue seems to be that of mass. According to the Standard Model, particle masses arise from the condensation of some field in the vacuum, analogous to the condensation mechanisms underlying superconductivity. In the original formulation of the model, it is a single, elementary, spinless field that condenses, leading to a single physical Higgs boson (named after Peter Higgs, a theorist who explored these possibilities in the 1960's). However, many physicists favour a composite Higgs field, by analogy with Cooper pairs. Unitarity arguments suggest that a Higgs boson should have a mass of less than about $1 \mathrm{TeV}$. Analyses of the high-precision LEP data give a preliminary indication that an elementary Higgs boson may be more likely to have a mass less than $300 \mathrm{GeV} / \mathrm{c}^{2}$ rather than greater than this value.

If the Higgs is indeed elementary, quantum corrections to its mass are uncontrollably large unless supersymmetric partners of all Standard Model particles exist, doubling the particle spectrum so that every fermion particle has a boson counterpart, and vice versa. In the minimal supersymmetric extension of the Standard Model there are three physical neutral Higgs bosons, as well as charged ones. At least one of the neutral ones is expected to have a mass similar to that of

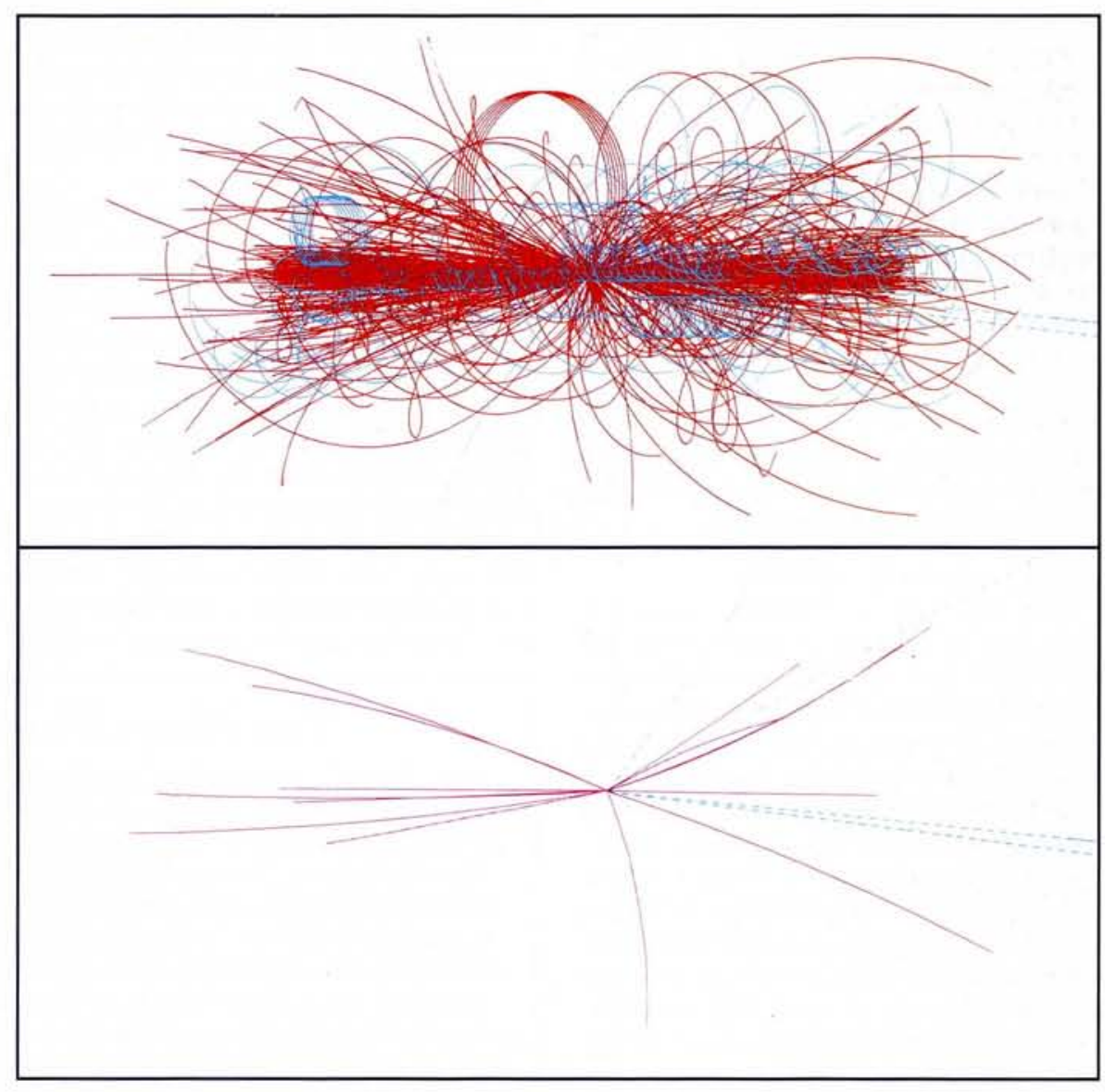

Fig. 1 - Upper: a computer simulation of all charged particles (hadrons in red, muons in blue) produced by 12 superimposed pp collisions occurring simultaneously during one bunch crossing which takes place every $15 \mathrm{~ns}$. The particle momenta transverse to the beam lines are typically of the order of $1 \mathrm{GeV} / \mathrm{c}$. A transverse momentum cut of $2 \mathrm{GeV} / \mathrm{c}$ therefore cleans the picture remarkably (lower figure) and the Higgs decay into four muons with high momenta becomes clearly visible $(8 \mathrm{~m}$ long $\times 4 \mathrm{~m}$ in diameter detector; $2 \mathrm{~T})$. 
the $Z$, probably in the range up to about $130 \mathrm{GeV} / \mathrm{c}^{2}$. Supersymmetry also helps to explain the very different strengths of the fundamental forces of physics. Theories of grand unification, in particular when combined with supersymmetry, provide accurate predictions for the ratios of gauge coupling strengths measured at LEP if the supersymmetric particles have masses smaller than about $1 \mathrm{TeV} / \mathrm{c}^{2}$ as expected on the basis of a priori theoretical arguments. Hence supersymmetry would produce a rich pattern of physics in the area accessible to the LHC.

\section{Top factory}

Although LEP has told us not to expect any more conventional quark or lepton generations, the top quark remains to be discovered. There could be a detailed interplay between the top quark and the Higgs sector. Regardless of whether the discovery of the top quark remains for the LHC, the machine will be a "top factory" producing very large numbers of top quark-antiquark pairs whose study should cast light on the problems of flavour.

\section{CP-violation}

One of the outstanding mysteries in physics and a key question within the Standard Model is CP-violation, observed 25 years ago in the kaon system. Perhaps linked to the predominance of particles over antiparticles in the Universe, CP-violation can be described, but not explained, by an empirical matrix (the so-called Cabibbo-Kobayashi-Maskawa matrix) of quark mixing parameters. It is expected that the CP-violation will find an echo in the $\mathrm{B}$ mesons. Physicists have so far been unable to detect enough of these mesons to observe CP-violation. However, B production rates at LHC, either in the collider mode, or with an internal jet target or an extracted beam, should open up this new domain, promising important new insights into a mysterious phenomenon.

\section{Heavy ions and ep collisions}

The LHC can also collide heavy ions (e.g., $\mathrm{Pb}$ ) to produce a total energy per nucleus of over $1250 \mathrm{TeV}$, thus entering a kinematic domain in such collisions which is unique and will not be accessible to any other machine for many years to come. The study of strongly interacting matter at these extreme energy densities $(8 \mathrm{GeV})$ $\mathrm{fm}^{3}$ ) and temperatures is of great importance. For instance, statistical $\mathrm{OCD}$ predicts that a transition from hadronic matter to a plasma of deconfined quarks and gluons will occur at these high densities - a transition which took place in the early Universe some $10^{-5}$ seconds after the Big Bang. Heavy ion experiments will allow the study of both the transition and the properties of the primordial quarkgluon plasma.

With LEP located in the same tunnel as LHC it is obvious to consider the possi- bility of colliding electrons on protons at centre-of-mass collision energies of 1.7 TeV. The reach in resolving power in ep collisions would be increased by a factor of 20 compared to the HERA accelerator presently being commissioned at DESY, Hamburg, with the first physics runs expected this summer.

\section{The Experimental Challenge}

Studying phenomena at the $1 \mathrm{TeV}$ mass scale requires high luminosities. Accordingly, an important characteristic of the LHC is its unprecedented high design luminosity $\left(>10^{34} / \mathrm{cm}^{2}\right.$ s and some $10^{4}$ larger than for existing colliders) implying a very hostile environment for the detectors. The luminosity and the narrow bunch structure (bunches of particles are separated by $15 \mathrm{~ns}$ ) mean that about 20 superimposed inelastic events are expected every $15 \mathrm{~ns}$ (Fig. 1). LHC detectors must therefore accept some 2000 particles per bunch crossing (more than 50000 particles in the case of heavy ion collisions). Consequently, the spatial resolution (or "granularity") of LHC experiments and hence the total number of electronic channels has to be increased by two orders of magnitude compared to the present LEP experiments. Furthermore, LHC detectors will have to withstand radiation levels far exceeding those previously encountered in high energy physics experiments. Some kilowatts of inelastic secondary particles are expected to be produced in each collision region.

With a total proton-proton cross-section of about 0.1 barns, the LHC design luminosity generates an event rate of some $10^{9} \mathrm{~Hz}$. However, processes with large constituent centre-of-mass energies, involving large transverse momentum particles or very heavy particles or both, are suppressed by at least a factor $10^{9}$, as can be deduced from Fig. 2 giving some characteristic cross-sections. Very sophisticated event triggers, with high efficiencies and very large rejection factors against unwanted events, have to be developed to reduce the final data rate to about
$100 \mathrm{~Hz}$. This task will be complicated even further by the extreme particle densities. The development of electronics working in the $\mathrm{GHz}$ region and capable of handling several hundred events per second will challenge designers.

To exploit the rich physics potential of the LHC, the experiments have to detect the various types of signatures which are expected. But they must also be sufficiently flexible to cope with the unexpected. A single detector is unlikely to be optimal for all of the likely signatures (see box). Moreover, the demanding experimental environment, the complex final states, and the rich variety of possible physics processes suggest that some redundancy and overlap between the capabilities of the different detectors for LHC is desirable.

\section{Towards the Experimental Programme}

The LHC experimental environment implies severe constraints on the detector components as regards radiation resistance, granularity and speed. It also demands a complicated trigger with several successive levels of triggering together with a highly complex and sophisticated data acquisition chain to extract the physics signals from the background. The requirements were reviewed at a workshop in Aachen in 1990 [1]. Many of the detector concepts which were presented for high luminosity proton experiments and for heavy ion and beauty experiments in collider or fixed-target modes have been pursued further via detailed simulations, design studies, and most importantly with a broad detector R. \& D. programme (see page 112). All are aware that technologies have to be pushed to a new level of performance so designs for LHC detectors should not be frozen too early.

A first important step in defining the LHC experimental programme was made at a meeting in Evian-les-Bains in March 1992 [2]. Twelve Expressions of Interest (EOI) for LHC experiments were presented and discussed for the first time, and three aditional EOI were received after the dead-

\section{The Main Observables at LHC}

Some signatures which LHC detectors should be capable of observing are:

Electrons and muons: detection with high efficiency and adequate energy resolution is vital, particularly for isolated leptons (e.g., for $\mathrm{H} \rightarrow \mathrm{ZZ} \rightarrow 4$ muons or electrons). Jets, the hadronization product of quarks and gluons, will be the building-blocks for hadronic final states: they should be measured accurately up to large repetition rates or, equivalently, down to small production angles.

Missing transverse energy due to invisible particles escaping the detector must be measured: the precision has to be comparable to the transverse energy resolution of typical individual charged leptons or jets. This requirement places a premium not only on an adequate energy resolution of the calorimeters, but also on the detector's hermeticity down to small forward angles.

Intermediate mass Higgs $\left(90-130 \mathrm{GeV} / \mathrm{c}^{2}\right)$ : photons should be measured with excellent energy and spatial resolutions to enable a search for the intermediate mass Higgs particle.

Short-lived heavy flavour particles, such as charm and beauty, must be tagged: a high-precision vertex detector will be needed. 


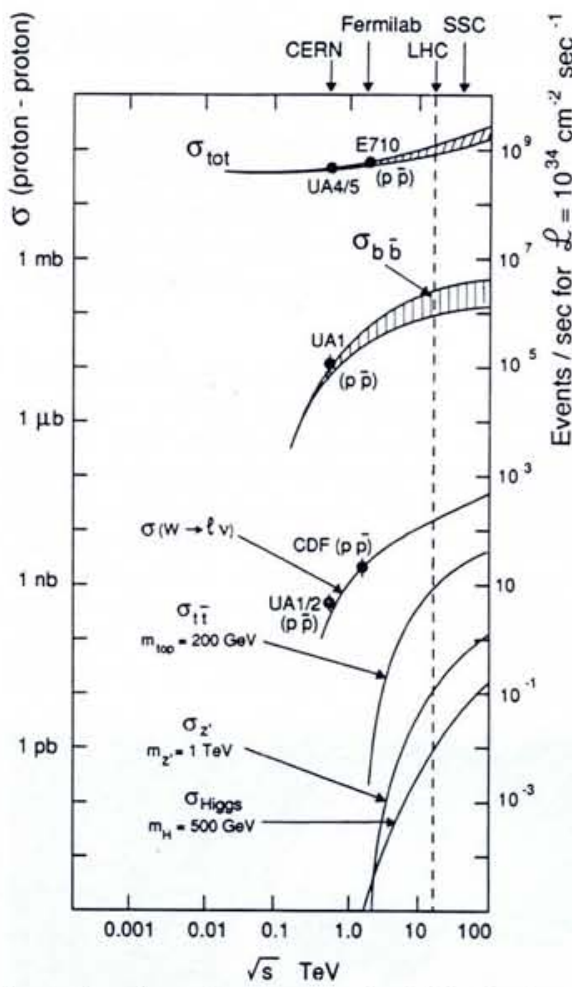

line. Together they cover the full physics potential of the LHC. The possible study of electron-proton collisions in LHC is being kept open for the moment pending the eagerly awaited first results from HERA that will hopefully indicate the research directions to follow. The wide variety of experiments covered by the EOI demonstrates the enthusiastic response to opportunities at LHC.

\section{Proton-proton detectors}

Four of the EOI's were concerned with large, general purpose, proton-proton collider detectors (ASCOT, CMS, EAGLE and $L 3+1)$ designed to study physics phenomena within and beyond the Standard Model at the highest LHC luminosity. At the same time they should be able to reap the harvest of interesting physics at lower luminosities, in particular in the top, beauty and tau sectors. As discussed on page 110, the four detectors adopt complementary experimental approaches using different detection techniques and magnet designs.

Large, powerful, detector magnets are needed for a precise muon momentum measurement over a large solid angle. Two basic choices for the field configuration were considered: a toroidal field with circumferential field lines around the particle beams and a solenoid configuration with the field lines parallel to the beams. So the four experiments essentially involve either toroids or solenoids, either with an open geometry, or filled with iron, often completed by toroids in the forward direction. The construction of these detector magnets, up to $12 \mathrm{~m}$ in radius and 30000 tonnes in weight, and their very large stored energies (up to $3 \mathrm{GJ}$, i.e., some 30 times larger than their LEP counter-
Fig. 2 - Energy dependence of some characteristic particle cross-sections $\sigma$, and event rates for a luminosity $L$ of $10^{34} / \mathrm{cm}^{2} s$. The production cross-section of the Higgs particle rises steeply from the CERN and Fermilab colliders (given by data points) to the LHC and the Superconducting Supercollider (SSC) now under construction in Texas, USA. However, it is still more than nine orders of magnitude below the total cross-section, not taking into account the branching ratio for the Higgs decay into, e.g., four muons, which is only $0.1 \%$. One of the challenges for the new colliders will be to trigger on these extremely rare events.

parts), presents a considerable technical challenge which can only be tackled efficiently using all of Europe's expertise in magnet construction.

\section{Heavy ion detectors}

The proposed study of heavy $(\mathrm{Pb})$ ion collisions is an unique physics opportunity which exploits in a natural way the existing CERN infrastructure and the expertise accumulated in accelerating oxygen and sulphur ions up to about $200 \mathrm{GeV} /$ nucleon at CERN's SPS machine ( $\mathrm{Pb}$ beams will be available in 1994). For heavy ion collisions, LHC would run at a modest luminosity $\left(2 \times 10^{27} / \mathrm{cm}^{2} \mathrm{~s}\right)$ and the experimental challenge is to handle the enormous multiplicities (some 50000 charged particles per event) in these interactions.

Three experimental set-ups were discussed at Evian for studying the quarkgluon-plasma (QGP) which is expected to be formed. One is a general-purpose detector, another considers the possible use of an existing LEP detector (DELPHI), and a third stresses the potential of one of the pp detectors (CMS).

To extract tell-tale signs of the QGP, the CMS heavy-ion option will concentrate on the measurement of muons, looking in particular for the production of heavy flavour bound states like the psi and upsiIon families, which are unique probes for the QGP. The dedicated detector proposed by the heavy ion community aims on the other hand to identify and measure all the soft particles in a central slice. The latest designs concentrate on a central cylindrical barrel detector, comprising either a very thin and small superconducting solenoid, or a larger solenoid with all the sub-detectors inside the coil. The detector components include a vertex and tracking system with several planes of high resolution detectors, a Time Projection Chamber (TPC) for pattern recognition and particle identification, a Time of Flight (TOF) and a Ring Imaging Cerenkov counter $(\mathrm{RICH})$ system to complement the TPC for particle identification, as well as a fine-grain electromagnetic calorimeter over a restricted solid angle for photon measurements.

\section{$B$ meson detectors}

High energy proton collisions are a prolific source of beauty particles making the LHC a possible alternative to dedicated beauty factories. So three of the $15 \mathrm{EOI}$ 's propose studying the production of $B$ mesons, the main objective being the observation of $\mathrm{CP}$-violation in the decays of B mesons. One EOI uses the LHC in a collider mode, the other two involve high energy protons in fixed target set-ups.

The collider detector specialised for beauty physics would run at relatively low luminosities $\left(<10^{32} / \mathrm{cm}^{2} \mathrm{~s}\right)$ to allow $\mathrm{mi}$ crovertex detectors to be operated near the proton beam. The main detector consists of a forward spectrometer with a 600 mrad aperture, $\mathrm{RICH}$ particle identification, electromagnetic calorimetry and a silicon microvertex detectors located in recesses in the vacuum pipe (so-called "Roman pots") to bring them as close as possible to the circulating beams. Sophisticated trigger processors reconstructing on-line the vertices of secondary B particles should make the acquisition and reconstruction of exclusive decays of $B$ particles of all types possible.

One of the two proposed, fixed-target beauty experiments studies the production of beauty mesons in the interactions of one of the LHC beams with an internal molecular cluster jet in a straight section, preferably near an existing LEP cavern. This thin production target (only $\pm 1 \mathrm{~mm}$ along the beam) greatly simplifies triggering based on the identification of a secondary vertex away from the primary interaction point. Some $10^{10}$ beauty pairs, selected using sophisticated triggers, would be produced within a year's operation. Microstrip detectors would again be located in Roman pots, while a RICH counter, an electromagnetic calorimeter and a muon identifier complete the detector.

The second fixed target beauty experiment would use a beam extracted from the LHC beam halo via a bent crystal - a channelling technique which has already been demonstrated at CERN and elsewhere, but which requires further development for this application.

\section{Neutrino detectors}

High energy proton-proton collisions in the LHC will generate a large flux of collimated high energy neutrinos (up to $1 \mathrm{TeV}$ ) in the very forward directions arising from the decay of heavy flavour particles. Two experiments therefore propose to use these neutrinos for muon- and electrontype neutrino interaction studies. The large tau-neutrino fraction $(10 \%)$ makes the observation and study of this still undiscovered neutrino an obvious experimental goal. The techniques to observe taus produced in tau-neutrino interactions are to a large extent based on those being prepared in two approved SPS experiments (CHORUS and NOMAD). Another 
method being considered for producing collimated, high energy neutrino beams is the use of a cesium gas jet traversing one of the circulating proton beams. A neutrino experiment could also be placed behind the LHC beam dump.

\section{Soft hadron detectors}

The three EOI received after the deadline for the Evian meeting last March present the obvious case for measuring total cross-section, elastic scattering and diffraction dissociation in $\mathrm{pp}$ interactions.

\section{Future Steps}

An important factor contributing to the progress made by the experimental community in understanding how to cope with the experimental challenge posed by the LHC is the Detector Research and Development programme under the aegis of CERN's Detector R. \& D. Committee (DRDC). About 1000 physicists from both CERN Member States and elsewhere are involved in the more than 25 projects presently approved and institutions from some 20 non-Member States collaborated in the EOI. This widespread interest in the LHC programme confirms CERN's rôle as a leading accelerator facility.

The procedure for approving experiments has been set in motion following the presentation of the EOI's in Evian. It has to reflect the fact that the LHC is primarily a discovery machine charting completely new physics territory. This is markedly different from the situation with LEP where the physics goals were clearly defined and the experimental solutions, although far from trivial, were well understood at the Letter of Intent stage.

The main lines towards the LHC experimental programme are therefore set. The intention is to establish within the next few months a LHC Committee (LHCC) to supervise the transition from the EOI's to Letters of Intent and, eventually, to technical proposals. The timetable is determined by the approval procedure of the LHC machine itself which, according to last December's CERN Council resolution, requires the definition as well as the costing of the basic programme by the end of 1993. Meanwhile, discussions are taking place within the two pairs of proto-collaborations involved in the large pp detectors (CERN has proposed two such experiments for LHC [3]). Building upon the fact that they share a common magnet geometry, i.e., toroids, ASCOT and EAGLE have agreed to submit a common Letter of Intent. CMS and $\mathrm{L} 3+1$ are expected to do the same for solenoids.

[1] Proc. Large Hadron Collider Workshop, Aachen; Eds G. Jarlskog and D. Rein, CERN Report 90-10 (1990).

[2] Proc. General Meeting on LHC Physics \& Detectors, Evian-les-Bains (CERN) 1992. [3] Design Study of the Large Hadron Collider, CERN Report 91-03 (1991).

\section{LHC's Proton-Proton Detectors}

Four high luminosity proton-proton collider detectors were presented as Expressions of Interest at the CERN/European Committee for Future Accelerators (ECFA) meeting in Evian-les-Bains in March 1992. ASCOT (Apparatus with SuperCOnducting Toroids) and EAGLE (Experiment for Accurate Gamma, Lepton and Energy Measurements) propose large magnetic toroids for the muon measurements; CMS (Compact Muon Solenoid) and $L 3+1$ (a modified version of the existing $L 3$ detector at $L E P$ ) seek large solenoids.

\section{ASCOT}

ASCOT (Apparatus with SuperCOnducting Toroids) has as first priority the precise measurement of muon momenta over a large range of solid angles without relying on central tracking. The magnet system consists of a superconducting air-cored toroid in the central region together with smaller iron-cored superconducting toroids in the forward regions. The toroids are instrumented with pressurised drift tubes with 100 micron resolution, resulting in a muon momentum resolution of $2 \%$ at $100 \mathrm{GeV}$ and $14 \%$ at $1 \mathrm{TeV}$ in the central region. Second priority is given to the uniformity and response stability of the electromagnetic calorimeter. The segmentation of the calorimeter aims to achieve the best possible identification of electrons and photons in a background dominated by hadrons. It is proposed to build a lead/liquid argon electromagnetic calorimeter with emphasis on lateral and

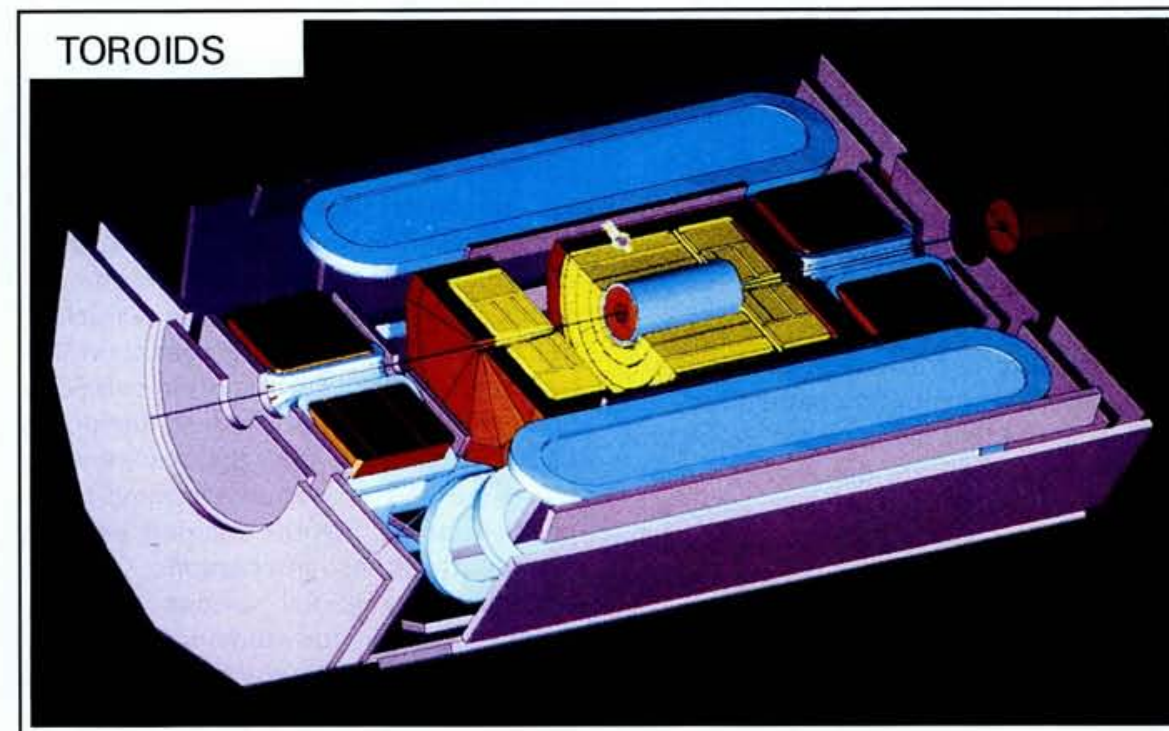

ASCOT

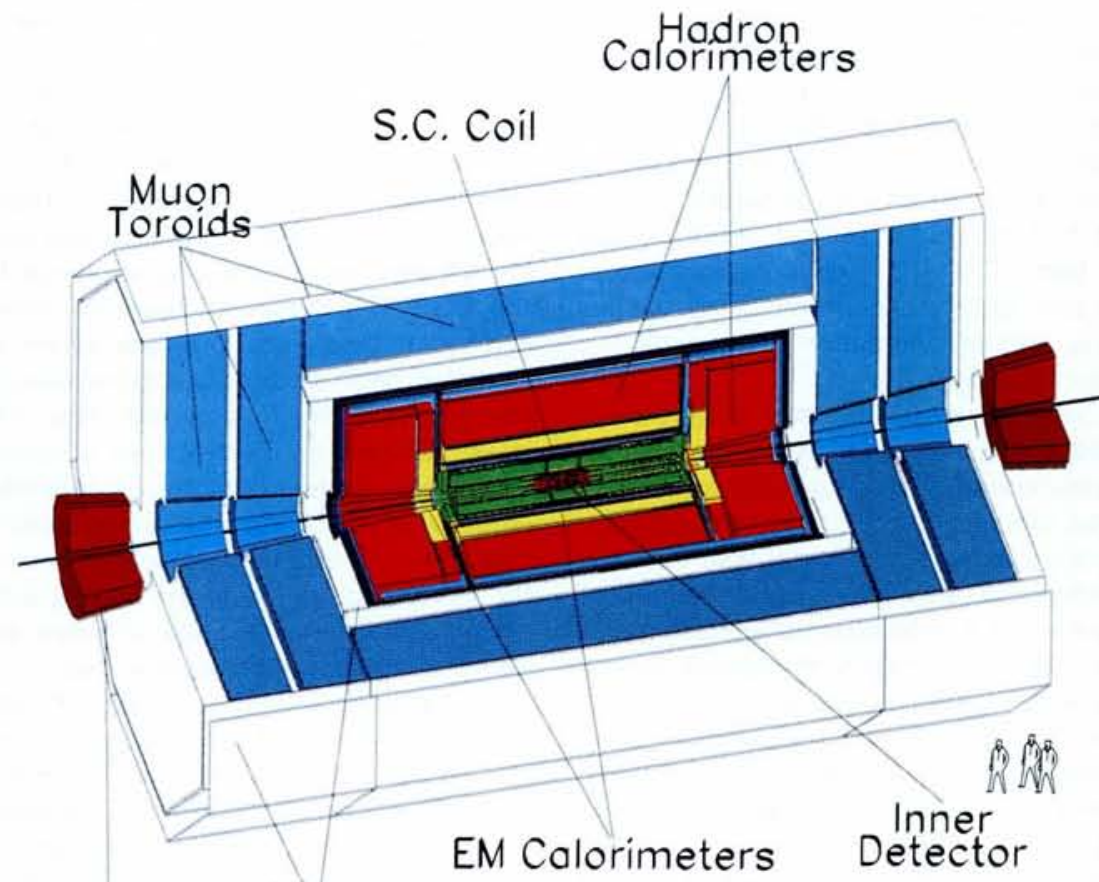

Forward

Calorimeters Detectors 